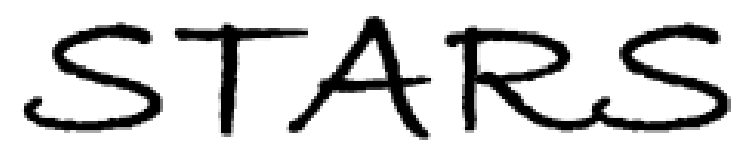

University of Central Florida

STARS

$1-1-2010$

\title{
Low-energy electron-impact ionization of the helium atom using the multiconfiguration Hartree-Fock method
}

Hari P. Saha

University of Central Florida

Find similar works at: https://stars.library.ucf.edu/facultybib2010

University of Central Florida Libraries http://library.ucf.edu

This Article is brought to you for free and open access by the Faculty Bibliography at STARS. It has been accepted for inclusion in Faculty Bibliography 2010 s by an authorized administrator of STARS. For more information, please contact STARS@ucf.edu.

\section{Recommended Citation}

Saha, Hari P., "Low-energy electron-impact ionization of the helium atom using the multiconfiguration Hartree-Fock method" (2010). Faculty Bibliography 2010s. 724.

https://stars.library.ucf.edu/facultybib2010/724

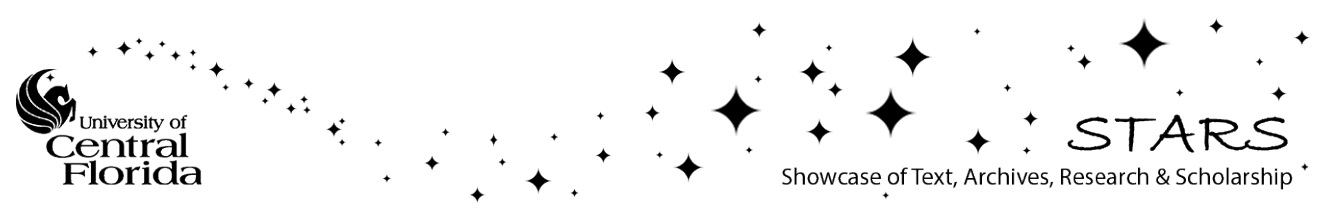




\title{
Low-energy electron-impact ionization of the helium atom using the multiconfiguration Hartree-Fock method
}

\author{
Hari P. Saha* \\ Physics Department, University of Central Florida, Orlando, Florida 32816, USA
}

(Received 30 June 2010; published 11 October 2010)

\begin{abstract}
We have extended the multiconfiguration Hartree-Fock method for electron-impact ionization of atoms to investigate the effects of polarization and electron correlation of the target in the initial state. As a test case, the method is applied to calculate triple differential cross sections for electron-impact ionization of He atom at an excess energy of $2 \mathrm{eV}$ for the coplanar $\theta_{12}=\pi$ geometry for equal and unequal energies of two final-state outgoing electrons. To determine the effects of electron correlation between the two outgoing electrons in the final state, the variationally determined screening potential approximation is used. It is found that target correlation in the initial state has smaller effect on the triple differential cross section but the polarization of the target by the incident electron has considerable effect on the triple differential cross section at low incident energy. The results are compared with absolute measurement and other available theoretical results.
\end{abstract}

DOI: 10.1103/PhysRevA.82.042703

PACS number(s): $34.80 . \mathrm{Dp}$

\section{INTRODUCTION}

The study of electron-impact ionization of atoms started several years ago because of its importance for understanding the dynamics of three charged particles interacting through Coulomb forces. A large number of experimental and theoretical data are available for triple differential cross sections at low and intermediate energies for a number of targets. Among them, hydrogen and helium have been studied in detail. The main emphasis has been on evaluating the final total wave function accurately, in particular, the electron correlation between the two outgoing continuum electrons which depends on how they share the excess energy as well as their angles of emission. Among several theoretical approaches, the $a b$ initio methods, the convergent close coupling (CCC) [1], the time-dependent close coupling [2], and the exterior complex scaling approaches [3] are successful in explaining the experimental data. The other approximate methods, the multichannel $R$-matrix theory [4], and the distorted wave (DW) methods [5], although less accurate, typically yield reasonably good agreement with the experimental results. Pan and Starace [6] used the distorted partial wave approach to study triple differential cross sections for hydrogen, helium, and also rare-gas elements. Brauner et al. [7,8] also studied triple differential cross sections for electron-impact ionization of $\mathrm{H}$ and $\mathrm{He}$ at low and intermediate energies using exact asymptotic wave functions. Temkin [9] developed a Coulomb-dipole theory to study the theoretical behavior of electron-impact ionization of atoms. On the experimental side, there have been several measurements at low excess energies. Among them the relative triple differentials for a He target were measured by Selles et al. [10] for equal energy sharing in which the two continuum electrons leave in opposite directions. Rosel et al. [11] measured the absolute triple differential cross section (TDCS) of He at low excess energy and for several angles of emission. Theoretically, only a few models have been applied to calculate

\footnotetext{
*hps1@ physics.ucf.edu
}

TDCSs for He at low energies. Pan and Starace [6] made a detailed analysis of TDCSs for $(e-2 e)$ processes in He and showed for the case in which the two electrons leave in opposite directions that the experimentally observed target dependence of near threshold TDCS essentially comes from short-range effects on the $s$-wave phase shifts of both incident and final-state continuum electrons. They reported results of DW calculations of TDCSs not only for $\mathrm{H}$ and $\mathrm{He}$ targets but also for rare-gas atoms $\mathrm{Ne}, \mathrm{Ar}, \mathrm{Kr}$, and $\mathrm{Xe}$. They compared their results with available experimental data and found them to be in very good agreement. Stelbovics et al. [12] calculated TDCSs for helium at several incident energies using the CCC approach. Their results for TDCSs for $2 \mathrm{eV}$ excess energy agreed very well with the experimental measurement of Rosel et al. [11] for the case in which two outgoing electrons were emitted in the opposite directions. Jones et al. [13] reported theoretical results of the TDCS for electron-impact ionization of He at $26.6 \mathrm{eV}$ energy using DW Born approximations. Crothers [14] obtained an approximate semiclassical solution of the Schrödinger equation for single scattering and calculated the TDCS for He. Colgan et al. [15] applied a close coupling time-dependent method to study electron-impact ionization of helium atoms. All these methods provide different accurate descriptions of the final-state wave functions. Recently, we have studied [16] TDCSs of $\mathrm{H}$ for $4 \mathrm{eV}$ excess energy shared both equally and unequally by the two final-state continuum electrons for $\theta_{12}=\pi$ geometry using Hartree-Fock (HF) [17] and the screening potential (SP) approximations [6,18,19]. Later we investigated [20] the application of the HF approximation to on the TDCS of $\mathrm{H}$ at several incident energies for the case in which two final-state continuum electrons leave in opposite directions.

As mentioned earlier, so far, considerable efforts have been made to determine the interaction between the two outgoing electrons in the final state. At low energy, in particular, close to threshold, electron correlation and polarization effects play an important role. It was shown earlier [21] that at low energy both polarization and electron correlation are very important in the scattering of electrons by the atoms. In the low-energy electron-impact ionization of atoms, the incoming and the 
outgoing electrons spend a great deal of time in the vicinity of the atom or the ion [22], resulting in the distortion of the atomic and ionic clouds. At low energies these polarization effects are, in general, allowed by assuming virtual excitations to the higher atomic states and must be treated accurately. To our knowledge no calculations are available which explicitly studied the effects of polarization and electron correlation of the initial state in the electron-impact ionization of atoms. It is discussed by Rosel et al. [11] that near threshold polarization of the target by the incident electron and the target correlation in the initial state might be important for electron-impact ionization of helium atoms. They also mentioned that to understand the physics involved in the angular distribution of electrons, one must include polarization and correlation of the target in the incident channel near threshold.

Inspired by the work of Rosel et al. [11], we have made a detailed investigation of the effects of target correlation and polarization on the electron-impact ionization of helium atoms. We consider the helium target as the ideal atom not only for its simple structure but for its well-known correlation and polarization. It has been shown [21] that the polarization effects at low energy can be taken into account very accurately completely ab initio by optimizing both continuum and excited bound orbitals simultaneously at each kinetic energy of the projectile. Recently, we have extended the multiconfiguration Hartree-Fock (MCHF) method of electron-impact ionization of atoms to investigate the effects of polarization and electron correlation of the target in the initial state on the TDCS of atoms. As a first application of the method, we carried out calculation of the TDCS of the He target for $2 \mathrm{eV}$ excess energy sharing equally and unequally by the two final-state outgoing electrons when they are leaving in opposite directions. The purpose of this calculation is to determine in the present configuration the effects of polarization and target correlation in the initial state on the TDCS at this low energy. The polarization and the electron correlation of the target in the initial state are considered completely ab initio in the MCHF approximation. The final-state correlation is included using the variationally determined SP $[6,18,19]$. We compared our TDCS results with the available experimental and theoretical data to determine the effects of polarization and electron correlation of the target on the electron-impact ionization of He atoms.

\section{THEORY}

The theory of the electron-impact ionization of helium atoms can be described by the MCHF method. The description of the method is given in our earlier articles [16,20]. Briefly, the TDCS for electron-impact ionization of atoms is given by [6]

$$
\frac{d^{3} \sigma}{d E_{2} d \Omega_{1} d \Omega_{2}}=\frac{(2 \pi)^{4}}{k} k_{1} k_{2}\left|\left\langle\Psi_{f}^{-}|V| \Phi_{i}^{+}\right\rangle\right|^{2},
$$

where $\vec{k}$ is the momentum of the incident electron and $\vec{k}_{1}$ and $\vec{k}_{2}$ are the momenta of the two continuum electrons in the final state. The solid angles $d \Omega_{1}$ and $d \Omega_{2}$ are associated with the two continuum electrons in the final state. $E_{i}=\frac{k_{i}{ }^{2}}{2}$ is the kinetic energy of the $i$ th final-state continuum electron. $\Phi_{i}^{+}$and $\Psi_{f}^{-}$ are respectively the initial- and the final-state wave functions of the system. The perturbation $V$ is defined approximately as [6]

$$
V=\sum_{i=1}^{N} \frac{1}{\left|r_{N+1}-r_{i}\right|}-V_{\mathrm{HF}}^{N+1}\left(r_{N+1}\right) .
$$

This term represents the difference between the exact Hamiltonian and the approximate Hamiltonian which is used to construct and describe approximately the initial-state $\Phi_{i}^{+}$. The first term describes the Coulomb interaction between the incident electron and the $N$ target electrons and the second term is a multiconfiguration $\mathrm{HF}$ approximation to this interaction which is used to construct and describe approximately the initial-state $\Phi_{i}^{+}$. The initial-state wave function $\Phi_{i}^{+}$is characterized by the orbital and spin angular momenta, $L_{0}$ and $S_{0}$, respectively, of the target and by the momenta $\vec{k}$ and the orbital angular momentum $l$ of the incident electron. The final-state wave function $\Psi_{f}$ is described by the orbital and spin angular momenta $L_{c}$ and $S_{c}$ of the $N-1$ electron of the core ion and by the momenta $\vec{k}_{1}$ and $\overrightarrow{k_{2}}$ and orbital angular momenta $l_{1}, l_{2}$ of the two continuum electrons.

Using the partial wave expansion for the incident electron and for each of the two final-state continuum wave functions, we expand the initial-state $\Phi_{i}^{+}$and the final-state $\Psi_{f}^{-}$wave function in terms of the antisymmetrized, $L S$ coupled wave function for the $(N+1)$-electron system. The TDCS then reduces to

$$
\sigma_{\mathrm{He}}^{(3)}=\frac{4 \pi}{k^{2}\left[L_{0}\right]\left[S_{0}\right]} \sum_{S}\left|\sum_{L}(2 L+1) A\left(L S \hat{k_{1}} \hat{k_{2}}\right)\right|^{2},
$$

where

$$
\begin{aligned}
& \begin{array}{l}
A\left(L S \hat{k_{1}} \hat{k_{2}}\right) \\
=\sum_{l_{1} l_{2}} \sum_{m_{1} m_{2}} i^{l+l_{1}+l_{2}} e^{i\left(\delta_{l}+\sigma_{l_{1}}+\delta_{l_{1}}+\sigma_{l_{2}}+\delta_{l_{2}}\right)}\left(\begin{array}{ccc}
l_{1} & l_{2} & L \\
m_{1} & m_{2} & 0
\end{array}\right) \\
\quad \times Y_{l_{1} m_{1}}\left(\theta_{1}, \phi_{1}\right) Y_{l_{2} m_{2}}\left(\theta_{2}, \phi_{2}\right)\left\langle\psi_{f}|V| \psi_{i}\right\rangle \\
\text { with }[x]=(2 x+1), \\
\qquad \psi_{i} \equiv \Psi_{i}\left(\left(L_{0} l\right) L_{T} M_{T}\left(S_{0} \frac{1}{2}\right) S_{T} M_{S_{T}}\right) \\
\psi_{f} \equiv \Psi_{f}\left(\left[L_{c}\left(l_{1} l_{2}\right) L\right] L_{T} M_{T}\left[S_{c}\left(\frac{1}{2} \frac{1}{2}\right) S\right] S_{T} M_{S_{T}}\right)
\end{array}
\end{aligned}
$$

Here $L$ and $S$ are the orbital and spin angular momenta of the final-state continuum pair and $L_{T} M_{T}$ and $S_{T} M_{S_{T}}$ are the total orbital and spin angular momenta of the system, respectively.

\section{A. Wave functions for the continuum electrons}

The description of the multichannel MCHF method for the bound and continuum wave functions was reported earlier [17]. We provide here a brief description of the method. The total wave function at energy $E$ and term value $L S$ is expressed as

$$
\Psi_{E}=\sum_{i=1}^{N_{i}} a_{i} \Phi_{i}\left(\gamma_{i} L_{i} S_{i} ; N\right) F_{k_{i} l_{i}}+\sum_{j=1}^{N_{j}} C_{j} \Phi_{j}\left(\gamma_{j} L_{j} S_{j} ; N+1\right) .
$$


The first term represents a wave function describing an $N$-electron target that is an eigenstate of $L_{i}$ and $S_{i}$ in terms of $N$-electron bound configuration states $\Phi_{i}\left(\gamma_{i} L_{i} S_{i} ; N\right)$ with configuration $\gamma_{i}$ and term $L_{i} S_{i}$, mixing coefficients $a_{i}$, and total energy $E_{i}$ coupled to a scattering wave function $F_{k_{i} l_{i}}$ with angular momentum $l_{i}$, to yield an antisymmetric configuration state for the $(N+1)$-electron system with final-term value $L S$ and configuration $\gamma_{j} k l$. In the second term $\Phi\left(\gamma_{j} L S ; N+1\right)$ are $(N+1)$-electron bound configurations which are eigenstates with the same $L$ and $S$ and which are included to allow for polarization and electron correlation effects. The preceding wave function is defined in terms of a set of radial functions $P_{i}(r), i=1, \ldots, m$. The set of radial functions describing the target is obtained from the MCHF bound-state calculation for the target and are kept fixed. The set of radial functions describing the continuum orbitals and the other excited bound orbitals are determined variationally. In the close coupling approach, these radial functions are the solutions of the integro-differential equations of the form [17]

$$
\begin{aligned}
& {\left[\frac{d^{2}}{d r^{2}}+\frac{2 Z}{r}-\frac{l_{i}\left(l_{i}+1\right)}{r^{2}}\right] P_{i}(r)} \\
& \quad=\frac{2}{r}\left[Y_{i}(r) P_{i}(r)+X_{i}(r)+I_{i}(r)\right]+\sum_{i^{\prime}} \epsilon_{i i^{\prime}} P_{i^{\prime}}(r),
\end{aligned}
$$

where $\frac{2}{r} Y_{i}(r)$ and $\frac{2}{r} X_{i}(r)$ are, respectively, direct and exchange potentials and $\frac{2}{r} I_{i}(r)$ represents interactions between the configurations. The off-diagonal parameters $\epsilon_{i i^{\prime}}$ are related to Lagrange multipliers that ensure orthogonality between the continuum and the bound electrons of the target having the same symmetry.

The bound radial functions satisfy the boundary conditions

$$
P_{i}(r) \underset{r \rightarrow 0}{\sim} r^{l_{i}+1}, \quad P_{i}(r) \underset{r \rightarrow+\infty}{\sim} 0 .
$$

Suppose there are $n_{a}$ linearly independent sets of solutions for $n_{a}$ number of open channels in the multichannel approximation. Let $\bar{P}_{i j}$ be the un-normalized radial wave function of the $i$ th channel corresponding to the $j$ th set; then the radial function $\bar{P}_{i j}$ satisfies the conditions

$$
\bar{P}_{i j}(r) \underset{r \rightarrow 0}{\sim} r^{l_{i}+1}, \quad \bar{P}_{i j}(r) \underset{r \rightarrow+\infty}{\sim} F_{i}(r) a_{i j}+G_{i}(r) b_{i j},
$$

where $a$ and $b$ are the coefficient matrices and $F_{i}(r)$ and $G_{i}(r)$ are, respectively, the regular and irregular Bessel or Coulomb functions depending on the target. If the target is an atom, then

$$
\begin{aligned}
F_{i}(r) & =\sqrt{\frac{2}{\pi k_{i}}} \sin \left(k_{i} r-\frac{l_{i} \pi}{2}\right), \\
G_{i}(r) & =\sqrt{\frac{2}{\pi k_{i}}} \cos \left(k_{i} r-\frac{l_{i} \pi}{2}\right) .
\end{aligned}
$$

If the target is an ion,

$$
\begin{aligned}
& F_{i}(r)=\sqrt{\frac{2}{\pi k_{i}}} \sin \left(k_{i} r-\frac{l_{i} \pi}{2}+\frac{q}{k_{i}} \ln 2 k_{i} r+\sigma_{l}\right), \\
& G_{i}(r)=\sqrt{\frac{2}{\pi k_{i}}} \cos \left(k_{i} r-\frac{l_{i} \pi}{2}+\frac{q}{k_{i}} \ln 2 k_{i} r+\sigma_{l}\right) .
\end{aligned}
$$

The energy-normalized channel wave function $\mathbf{P}$ subjected to the preceding boundary condition can be written as

$$
\mathbf{P}=\overline{\mathbf{P}} \mathbf{C},
$$

where $\mathbf{C}$ is the normalization matrix,

$$
\mathbf{C}=(a+i b)^{-1} \text {. }
$$

If $\bar{\Psi}_{i}$ is the $i$ th solution of the un-normalized final-state wave function, then the $j$ th normalized final-state wave function is

$$
\Psi_{j}=\sum_{i} \bar{\Psi}_{i} C_{i j} .
$$

In the present case the radial function for the initial-state continuum wave function is calculated in the three different approximations. In the first, we use a HF approximation where the polarization and electron correlation of the target are ignored. In the second we included the target correlation in the MCHF approximation through the configuration interaction procedure and calculated the continuum wave function in the presence of the correlated target. Third, the radial wave function for the initial-state continuum wave function is calculated completely ab initio by including configurations which represent polarization. The final-state continuum electron radial wave function is calculated in the two approximations. First, we use the HF approximation where the polarization and electron correlation between the two continuum electrons in the final state are neglected. In this case each of the continuum electron wave functions is calculated in the potential of $\mathrm{He}^{+}$ion core. Second, the SP approximation $[6,18,19]$ is used to calculate the final-state continuum radial wave function. In this approximation, the exact Coulomb interaction between the two continuum electrons in the final state is replaced by a variationally determined SP.

\section{COMPUTATIONAL PROCEDURE}

In this article our focus is on calculating accurately the initial-state wave function and determining its effects on the electron-impact ionization of helium atoms.

\section{A. Initial state}

First we calculated the bound-state wave function of the target helium atom in the HF approximation. Then, using this wave function, the correlated wave function of the target is calculated by the MCHF wave function expansion with the excited orbitals coupled to form the ${ }^{1} S$ term. The excited orbitals considered are $2 s, 2 p, 3 s, 3 p$, and $3 d$. The calculated energy is found to be -2.901681 a.u., which is in very good agreement with the most accurate value -2.9037438 a.u. [23]. The continuum wave function in the initial state is then calculated in the HF approximation with the HF wave function of the target helium atom, as well as in the potential of the correlated target using the MCHF approximation at the initial incident energy $E_{0}=26.6 \mathrm{eV}$ for angular momentum $l=0$ to $l=6$.

As stated earlier, polarization of the $1 s^{2}$ target atom by the projectile is very important at this low-energy $e$-He scattering. This electron polarization is considered in the expansion of the initial-state continuum wave function through the 
$(N+1)$-electron excited bound configurations in the second term of the continuum wave function expansion [Eq. (5)]. The configurations which represent the dipole polarization are generated by the single replacement of the $1 s$ orbital of the target with orbitals representing the dipole polarization effects. In this case the polarization effects have been taken into account by the excited bound configurations generated by the replacement $1 s \rightarrow n p, n \leqslant 5$. All the configurations generated in this way are retained in the expansion of the initial-state continuum wave function. These excited bound orbitals which are responsible for the polarization of the target atom are determined by optimizing both the excited bound and the continuum orbitals $k l$ simultaneously at the kinetic energy of the projectile for partial waves $l=0-6$. This procedure, which includes the polarization of the target atom in an $a b$ initio manner, reproduces this effect more accurately [21]. The present calculation converges very well for all the partial waves considered in this case. The wave functions and the phase shifts calculated in this way are then used to calculate the matrix elements required to calculate the TDCSs.

\section{B. Final state}

The final-state continuum electron wave functions are calculated in the HF and the SP approximations $[6,18,19]$. In the HF approximation, each of the final-state continuum electron wave functions is calculated in the potential of the singly charged $\mathrm{He}^{+}$ion. In the SP approximation the exact Coulomb interaction between the two continuum electrons in the final-state $\Psi_{f}^{-}$is replaced by a variationally determined SP due to the mutual screening interactions. For the present configuration in which the two final-state electrons are leaving in the opposite direction, the SP for the two continuum electrons are determined by the effective screening charges $\Delta_{1}$ and $\Delta_{2}$ which are obtained by the condition $[6,18,19]$

$$
\frac{Z_{T}-\Delta_{1}}{k_{1}}+\frac{Z_{T}-\Delta_{2}}{k_{2}}=\frac{Z_{T}}{k_{1}}+\frac{Z_{T}}{k_{2}}-\frac{1}{k_{1}+k_{2}},
$$

where $Z_{T}$ describes the net asymptotic charge of the ionized target. The effective screening charges which satisfy the preceding relation are obtained as $[6,19]$

$$
\Delta_{i}=\frac{k_{i}^{2}}{\left(k_{1}+k_{2}\right)^{2}} \quad(i=1,2) .
$$

The wave functions for each of the final-state continuum electrons are calculated in both the HF and the SP approximation at both equal $E_{1}=E_{2}=1 \mathrm{eV}$ and unequal energy $E_{1}=1.5$ and $E_{2}=0.5 \mathrm{eV}$ for the angular momentum from $l=0$ to $l=6$ for each partial wave $L S$. In each of these cases the continuum wave function is made orthogonal to the bound orbitals of the target or the $\mathrm{He}^{+}$ion core having the same angular momentum $l$. The TDCSs are then calculated with the three sets of initial-state wave functions and the two sets of final-state wave functions for equal and unequal energy sharing between the two final-state electrons. The convergence with respect to the number of angular momentum $\left(l_{1}, l_{2}\right)$ pairs included in each partial wave $L$, as well as convergence with respect to the total number $L$ partial wave, were tested and it is found that partial waves $L=0-6$ are sufficient to obtain convergence in the cross section.

\section{RESULTS}

As already mentioned, the purpose of the present investigation is to determine the contribution of the effects of polarization and correlation of the target in the initial state on the TDCS for the electron-impact ionization of helium atom. In Fig. 1 we present the TDCS of helium for the case when the two outgoing electrons share the $2 \mathrm{eV}$ excess energy equally and leave in the opposite directions as a function of $\theta_{1}$. The initial state is calculated in three different approximations: (i) HF approximation where electron correlation and polarization of the target are neglected; (ii) polarization approximation (POL), where the polarization of the target due to the incoming projectile is included; (iii) electron correlation of the target (TCOR), which is included through the configuration interaction procedure. The final state is calculated in the HF approximation where the electron correlation between the two outgoing electrons is neglected. We compared our present results with experimental [11] and the theoretical results of Stelbovics et al. [12] calculated in the CCC approximation and the results obtained by Jones and Madison [11] using the DW approximation. The difference of the HF-HF from the TCOR-HF and the POL-HF results shows the contribution of the target correlation and the polarization. It is seen from the figure that the results obtained in the HF approximation and with target correlation are very close to each other for all ranges of $\theta_{1}$ except around the local maximum at $90^{\circ}$. For the range of $\theta_{1}$ from $60^{\circ}$ to $120^{\circ}$, the $\mathrm{HF}-\mathrm{HF}$ results are in excellent agreement with experiment [11] and the CCC calculation [12], whereas the TCOR-HF results are lower. On the other hand, it is interesting to see that the polarization effect has made a larger contribution than the correlation in the target. The polarization lowers the cross section and brings the cross section closer to the experimental result [11] and the DW calculation. These results also show maximum at $\theta_{1}=90^{\circ}$ in agreement with experimental and other theoretical results but at this angle it is still lower than that seen in the experiment [11]. Comparison shows that among the three approximations in the initial state the POL-HF results are in overall better agreement with experimental [11] and the

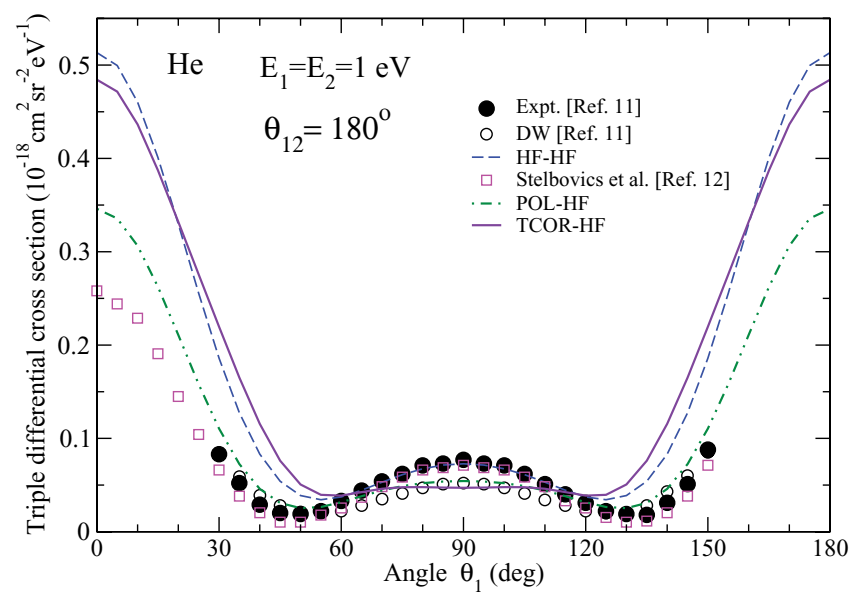

FIG. 1. (Color online) Comparison of TDCSs of He for $\theta_{12}=\pi$, $E_{0}=26.6 \mathrm{eV}$, and equal energy sharing with the initial state calculated in the three approximations and the final state calculated in the HF approximation. 


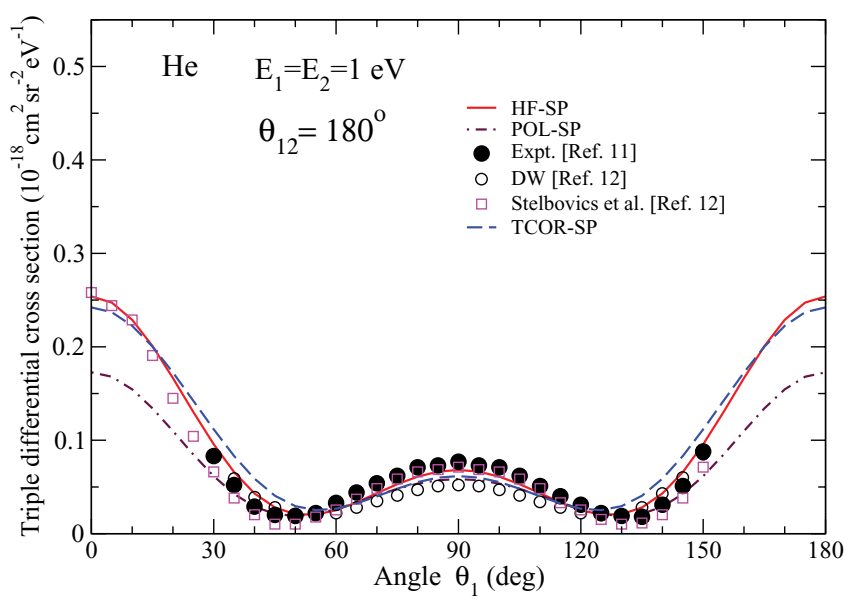

FIG. 2. (Color online) Same as Fig. 1, but with the final state calculated in the screening potential approximation.

CCC data [12]. This indicates that the effect of polarization in the initial state is much more important than the target correlation in the initial state. Both results are higher than the experimental [11] as well as theoretical results obtained in the CCC [12] and DW approximations [11] for $\theta_{1}<50^{\circ}$ and $\theta_{1}>$ $130^{\circ}$. These results are slightly lower than the experimental and the CCC results for $75^{\circ}<\theta_{1}<105^{\circ}$, whereas they are close to DW results [11] for this range of $\theta_{1}$.

The present results obtained with three different approximations in the initial state and the final state calculated in the SP approximation are presented in Fig. 2. The experimental [11] and the theoretical results obtained with CCC [12] and DW approximations [11] are included for comparison. As described earlier, in the SP approximations the exact Coulomb interaction between the two final-state continuum electrons are replaced by a variationally determined potential due to the mutual screening interaction using effective charges which satisfy the proper asymptotic boundary conditions $[6,18,19]$. The differences of the TCOR-SP and the POL-HF results from the HFSP approximation show respectively the contribution of target correlation and the polarization of the target in the incident channel. Compared to the HF-SP calculation, the target correlation increases the cross section except for $60^{\circ}<\theta_{1}<120^{\circ}$, whereas polarization decreases the cross section. All the results show a local maximum at $\theta_{1}=90^{\circ}$. It is seen from the figure that the effect of polarization is larger compared to target correlation. The present results with the initial state in the HF approximation agree very well with experiment and the CCC results of Stelbovics et al. [12]. These results also agree well with the DW cross sections except for $75^{\circ}<\theta_{1}<110^{\circ}$, where the DW results are slightly lower. It should be mentioned that Pan and Starace [6] made calculations similar to the present HF-SP calculation. Their cross sections are in excellent agreement with our HF-SP results. The present results obtained including electron correlation in the target (TCOR-SP) in the initial state are higher than the experimental, CCC, and DW results for $\theta_{1}<55^{\circ}$ and for $\theta_{1}>125^{\circ}$, but for $60^{\circ}<\theta_{1}<$ $125^{\circ}$ they agree very well with experiment and the CCC results; the DW results are slightly lower. The present results with polarization (POL-SP) in the initial state agree very well with experimental and the CCC results except for $\theta_{1}$ between

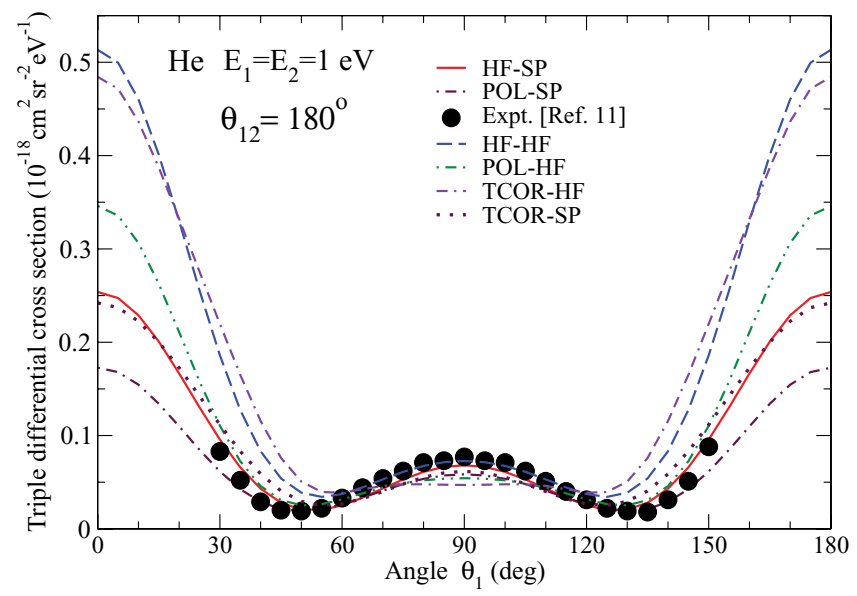

FIG. 3. (Color online) Same as Fig. 1, but with the final state calculated in the HF as well as screening potential approximation.

$75^{\circ}$ and $105^{\circ}$, where it is slightly lower. These results agree very well with the DW calculation. It is unclear why the HF-SP results without polarization and target correlation are in excellent agreement with experimental results, although there is considerable contribution due to polarization of the target in the initial state. It raises a question about the accuracy of the SP used to account for final-state correlation.

In Fig. 3, we present the TDCSs obtained with the initial state in the three different approximations and the final state in the two different approximations for the case in which two final-state electrons share $2 \mathrm{eV}$ excess energy equally and the relative angle between the two is $\theta_{12}=180^{\circ}$ as a function of $\theta_{1}$. The experimental [11] results are included for comparison. Comparison of the TCOR-HF and the TCOR-SP cross sections shows that the final-state correlation lowers the cross section for $\theta_{1}<60^{\circ}$ and for $\theta_{1}>120^{\circ}$. For $60^{\circ}<\theta_{1}<$ $120^{\circ}$ it increases the cross section, making the cross section closer to experiment. Similar behavior is also observed when comparison is made between the POL-HF and the POL-SP cross sections. From the figure it is seen that the present results obtained with the initial state in the HF approximation and the final state with SP are in excellent agreement with experimental results. In addition to the HF-SP results, the POL-SP and TCOR-SP results are very close to experimental results. The present results in three different approximations in the initial state and the final state in the HF approximation clearly show the contribution of the electron correlation and the polarization in the initial state. The target correlation effects increase the cross section, whereas the target polarization decreases the cross section except for $\theta_{1}$ around $90^{\circ}$, where both cross sections are lower than the HF-SP results. It appears that the results obtained including correlation and polarization in the initial state and the SP in the final state are closer to the experimental and the CCC results compared to those with final state in the HF approximation. Comparison of six different results shows that, besides the HF-SP results, which agree very well with experimental results, the present results obtained with target correlation and the polarization in the initial state and the SP in the final state, and also the results with polarization in the initial state and the final state without correlation, are very close to experimental results. 


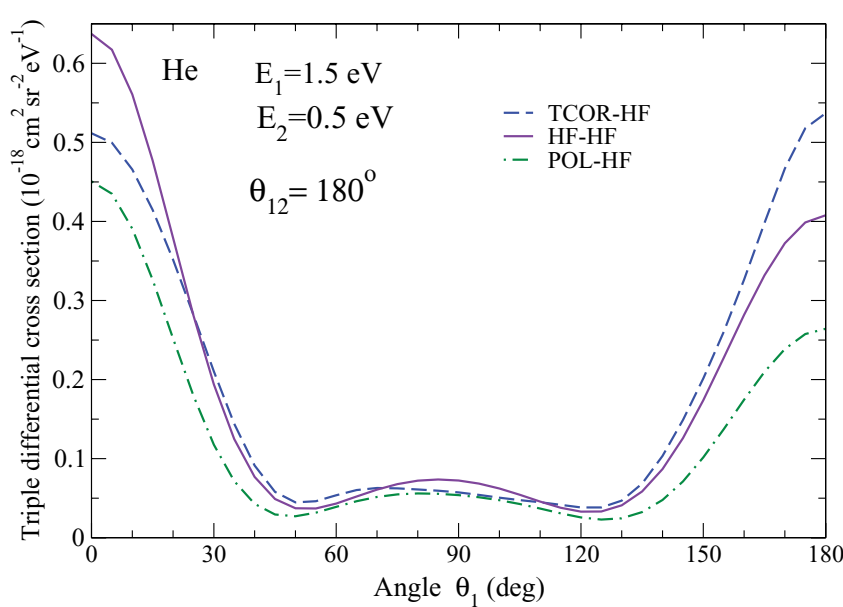

FIG. 4. (Color online) Same as Fig. 1, but for unequal energy sharing.

The present calculation shows that both electron correlation and polarization effects in the initial state are important.

In Fig. 4, we present our results for TDCS for unequal sharing of $2 \mathrm{eV}$ excess energy between the two final-state outgoing electrons as $E_{1}=1.5 \mathrm{eV}$ and $E_{2}=0.5 \mathrm{eV}$ in which $\theta_{12}=\pi$ as a function of $\theta_{1}$. The initial state is calculated in three different approximations as in the equal-energy case. The final state is calculated in the HF approximation where correlation between the two electrons are neglected. Comparison between the HF-HF and the TCOR-HF results shows the contribution due to the target correlation, which is small compared to that due to the effects of polarization as seen from the difference between the HF-HF and the POL-HF cross sections. Again, in the case of unequal energy sharing, the polarization lowers the cross section similarly to the equal-energy case. The results obtained with the HF and the TCOR approximations in the initial state are very close to each other for $20^{\circ}<\theta_{1}<160^{\circ}$. For $\theta_{1}<20^{\circ}$ the HF results are higher than the TCOR cross sections, whereas for $\theta_{1}>160^{\circ}$ the TCOR results are higher. The results obtained including polarization are close to $\mathrm{HF}$ and TCOR results for $50^{\circ}<\theta_{1}<130^{\circ}$. For $\theta_{1}<50^{\circ}$ and $\theta_{1}>130^{\circ}$ the polarization

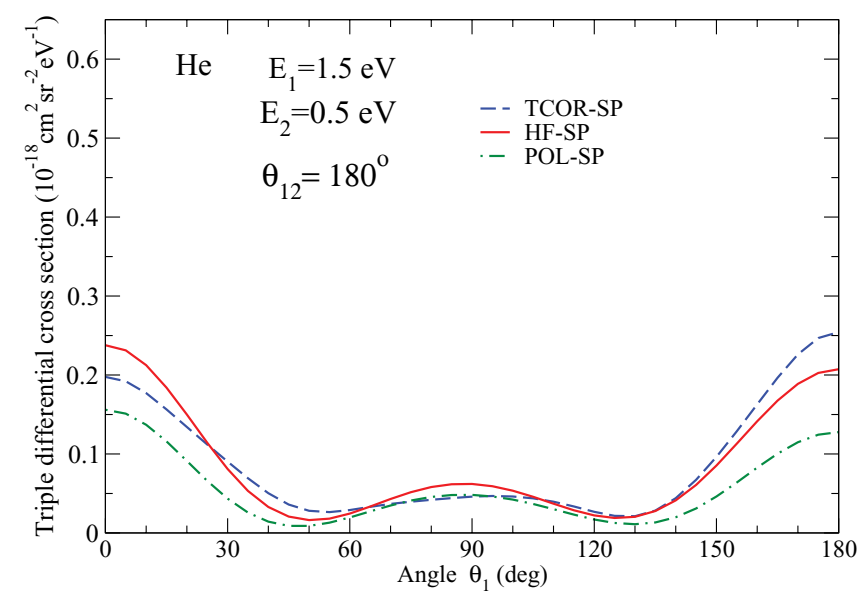

FIG. 5. (Color online) Same as Fig. 2, but for unequal energy sharing.

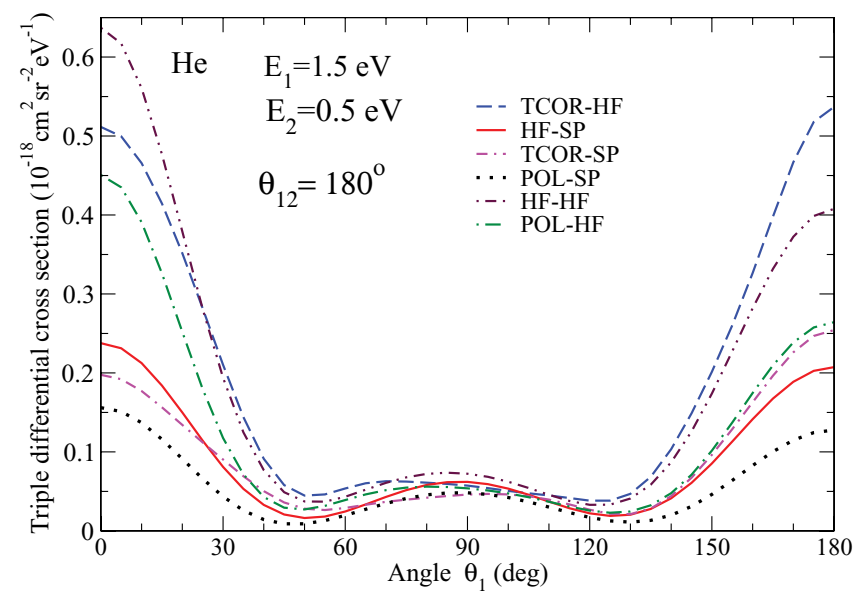

FIG. 6. (Color online) Same as Fig. 3, but for unequal energy sharing.

results are lower. It is similar to the results described in Fig. 1 for equal energy sharing by the two outgoing electrons.

We presented results of TDCSs with the initial state calculated in three different approximations and the final state calculated in the SP approximation which includes the correlation between the two final-state outgoing electrons with unequal energies $E_{1}=1.5 \mathrm{eV}$ and $E_{2}=0.5 \mathrm{eV}$ in Fig. 5. Again, in this case the HF-SP and the TCOR-SP cross sections are close to each other, whereas the polarization results are also close for $50^{\circ}<\theta_{1}<130^{\circ}$. For other angles of $\theta_{1}$ the polarization results are lower than the HF-SP and the TCOR-SP cross sections. In this case also with the inclusion of final-state correlation the qualitative feature of the cross sections obtained in the three different approximations in the initial state is the same as in the equal-energy case. The cross sections calculated in the HF-SP and POL-SP approximations have local maximum at $\theta_{1}=90^{\circ}$, whereas the TCOR-SP cross section has local maximum at $\theta_{1}=100^{\circ}$.

In Fig. 6, the results of TDCS obtained with the initial state in three different approximations and the final state calculated in the two different approximations are presented for the final state outgoing electrons sharing unequal energies $E_{1}=1.5 \mathrm{eV}$ and $E_{2}=0.5 \mathrm{eV}$. We assume that the HF-SP results are better as evidenced in the case of the equal-energy-sharing case. All the results are close to each other for $50^{\circ}<\theta_{1}<130^{\circ}$ except for the HF-HF results, which are slightly higher. This figure compares the contribution of electron correlation and polarization in the initial state. In general, polarization results are always lower than those with and without the contribution of final-state correlation at almost all angles of $\theta_{1}$ considered here.

\section{CONCLUSION}

The multiconfiguration HF method for bound and continuum wave functions has been applied to calculate TDCS for electron-impact ionization of helium atoms. The electronimpact ionization of helium atoms is a simple but an ideal case for determining the effects of electron correlation and polarization of the target on the TDCS. In this article we have investigated in detail the effects of electron correlation and polarization of the helium target in the initial state using 
the recently extended MCHF method. Calculation is carried out for incident electron energy $E_{0}=26.6 \mathrm{eV}$ and for both equal $E_{1}=E_{2}=1 \mathrm{eV}$ and unequal $E_{1}=1.5 \mathrm{eV}$ and $E_{2}=$ $0.5 \mathrm{eV}$ sharing of $2 \mathrm{eV}$ excess energy for the coplanar $\theta_{12}=\pi$ geometry. We presented the results for TDCSs with the initial state calculated in the three different approximations: (i) the HF approximation, (ii) including electron correlation in the initial target (TCOR), and (iii) including polarization of the target in the initial state by the incident electron. The final state is calculated in the two approximations: (i) the HF approximation (HF) in which each of the final-state continuum electron wave functions is calculated in the presence of a $\mathrm{He}^{+}$core, and (ii) the SP approximation $[6,18,19]$. We compare our results with available experimental and theoretical results. We found that in the present configuration the electron correlation of the initial target state has smaller effect on the TDCS than the electron polarization of the target in the initial state. Comparison shows that the electron polarization effect lowers the TDCS for all ranges of $\theta_{1}$. In the equal-energy case the present results with initial state calculated in the HF approximation and the final state calculated in the SP approximation are in excellent agreement with experiment of Rosel et al. [11] and the theoretical results of Stelbovics et al. [12] calculated in the CCC approach. The results obtained by Rosel et al. [11] calculated in the DW approximation are also close to these results. Although our calculation shows that considerable contribution comes from the polarization in the initial state, our observation shows that the HF-SP results without polarization in the initial state are in better agreement with experiment than those obtained including polarization. This raises a question about the SP approximation accounting for the final-state correlation. To answer this question, one should include the correlation in the final state accurately and completely $a b$ initio, just as the polarization was considered completely $a b$ initio in this article. We emphasize that the justification for publishing this article is only to test the extended MCHF method for $\mathrm{He}$ for both equal and unequal energy cases. In this article we have presented results showing effects of electron correlation and polarization of the initial state on the TDCS of the helium atom using the MCHF method for the case when two final-state electrons are leaving in the opposite direction. In the near future we plan to include electron correlation between the two final-state outgoing electrons completely ab initio in the MCHF approximation.

\section{ACKNOWLEDGMENTS}

I am grateful to A. F. Starace for many valuable discussions and Igor Bray for sending me numerical data. I thank Don Madison for reading the manuscript.
[1] I. Bray, J. Phys. B 33, 581 (2000).

[2] J. Colgan, M. S. Pindzola, F. J. Robicheaux, D. C. Griffin, and M. Baertschy, Phys. Rev. A 65, 042721 (2002).

[3] T. N. Resigno, M. Baertschy, W. A. Isaacs, and C. W. McCurdy, Science 286, 2474 (1999).

[4] K. Bartschat and P. G. Burke, J. Phys. B 20, 3191 (1987).

[5] D. H. Madison, R. V. Calhoun, and W. N. Shelton, Phys. Rev. A 16, 552 (1977).

[6] C. Pan and A. F. Starace, Phys. Rev. Lett. 67, 185 (1991); Phys. Rev. A 45, 4588 (1992).

[7] M. Brauner, J. S. Briggs, and H. Klar, J. Phys. B 22, 2265 (1989).

[8] M. Brauner, J. S. Briggs, H. Klar, J. T. Broad, T. Rosel, K. Jung, and H. Ehrhardt, J. Phys. B 24, 657 (1991).

[9] A. Temkin, Phys. Rev. Lett. 49, 365 (1982).

[10] P. Selles, A. Huetz, and J. Mazeau, J. Phys. B 20, 5195 (1987).

[11] T. Rosel, J. Roder, L. Frost, K. Jung, H. Ehrhardt, S. Jones, and D. H. Madison, Phys. Rev. A 46, 2539 (1992).
[12] A. T. Stelbovics, I. Bray, D. V. Fursa, and K. Bartschat, Phys. Rev. A 71, 052716 (2005).

[13] S. Jones, D. H. Madison, and M. K. Srivastava, J. Phys. B 25, 1899 (1992).

[14] D. S. F. Crothers, J. Phys. B 19, 463 (1986).

[15] J. Colgan, M. S. Pindzola, G. Childers, and M. A. Khakoo, Phys. Rev. A 73, 042710 (2006).

[16] H. P. Saha, J. Phys. B 41, 055201 (2008).

[17] H. P. Saha and D. J. Murray, J. Phys. B 38, 3015 (2005).

[18] M. R. H. Rudge and Seaton, Proc. R. Soc. London A 283, 262 (1965).

[19] S. Jetzke, J. Zaremba, and F. H. M. Faisal, Z. Phys. D 11, 63 (1989); F. H. M. Faisal, in Atoms in Strong Fields, edited by C. A. Nicolaides, C. W. Clark, and M. H. Nayfeh (Plenum, New York, 1990), p. 407.

[20] H. P. Saha, Phys. Rev. A 77, 062705 (2008).

[21] H. P. Saha, Phys. Rev. Lett. 65, 2003 (1990).

[22] A. Temkin, Phys. Rev. 107, 1004 (1957).

[23] K. Frankowski and C. L. Pekeris, Phys. Rev. 146, 46 (1966). 